\title{
Defining HRTEM Resolution: Image Resolutions and Microscope Limits
}

\author{
Michael A. O'Keefe, * Lawrence F. Allard** and Douglas A. Blom*** \\ *OKCS, 18528 Mesa Verde Way, Castro Valley, CA 94552 \\ ** Materials Science \& Technology Div., Oak Ridge National Lab, Oak Ridge, TN 37831 \\ ***EM Center, University of South Carolina, 715 Sumter St, Columbia, SC 29208
}

The high-resolution transmission electron microscope (HRTEM) is characterized by resolution and information limit [1]. Within these absolute instrumental limits, any particular image may experience additional restrictions on resolution due to non-optimal operating conditions, environmental factors, or the characteristics of the specimen under investigation. An individual HRTEM image (micrograph) may also contain additional non-linear (fringe or second-order) content $[2,3]$.

The microscope information limit (MIL) is the limit on the specimen's structural information that the microscope can deliver to the image. The microscope resolution limit (MRL or, more commonly, "microscope resolution") is the limit on information that can be delivered by the microscope to the image with same-sign phase [4]. MIL and MRL are designated microscope "limits" since they set upper bounds for the microscope, but "fringe resolution" is a non-limiting image artifact measured from high-frequency spacings generated in the image by the physics of transfer from the wave amplitude at the specimen exit surface to the wave intensity recorded at the image plane. The highestfrequency spacing present in the image may be used to demonstrate "fringe resolution", but is not a measure of either the MRL or the MIL unless the spacing can be shown to be present in the image via linear transfer from the specimen [2]. The MRL cannot exceed the MIL.

HRTEM resolution is the ability to distinguish between two atoms (or atom columns) in real (image) space; MRL can be investigated by using series of test objects to obtain micrographs showing closely-spaced atom columns [3]. Resolution quality can then be measured to refine the positions of atoms from image peaks [3]. Reciprocal-space frequencies corresponding to the atom spacing must be present in the test image diffractogram to verify resolution, but higher diffractogram frequencies that do not correspond to real-space separations cannot be labeled "resolution" [1]. Imaging theory can explain formation of the image plane intensity from specimen exit surface wave amplitude [1-3]. Image intensity, $\mathrm{I}(\mathbf{x})=\psi(\mathbf{x}) \cdot \psi^{*}(\mathbf{x})$, gives an image intensity spectrum $\mathrm{I}(\mathrm{u})=\Psi(\mathrm{u}) \odot \Psi^{*}(-\mathrm{u})$, where $\odot$ represents convolution and $\Psi(\mathbf{u})=\mathcal{F}\{\psi(\mathbf{x})\}$ is the FFT of $\psi(\mathbf{x})$. Limited temporal and spatial coherence (focus spread $\Delta$ and beam convergence $\alpha$ ) produce a transmission cross-coefficient (TCC) of $D\left(u^{\prime}, u^{\prime}-u\right)=B\left(u^{\prime}, u^{\prime}-u\right) . C\left(u^{\prime}, u^{\prime}-u\right)$, where $B\left(u^{\prime}, u^{\prime}-u\right)=\exp \left\{-1 / 2 \pi^{2} \lambda^{2} \Delta^{2}\left(u^{\prime 2}-\left(u^{\prime}-u\right)^{2}\right)^{2}\right\}$ is the TCC due to limited temporal coherence; $C\left(u^{\prime}, u^{\prime}-u\right)=\exp \left\{-\pi^{2} \alpha^{2}\left[\varepsilon u+C_{S} \lambda^{2}\left(u^{\prime 3}-\left(u^{\prime}-u\right)^{3}\right)\right]^{2}\right\}$ is the TCC due to limited spatial coherence; here $\lambda$ is electron wavelength, $\varepsilon$ is defocus, and spherical aberration coefficient is $\mathrm{C}_{\mathrm{S}}$ [2]. The image intensity spectrum is then $I_{\alpha \Delta}(u)=\sum_{u^{\prime}} \Psi\left(u^{\prime}\right) \cdot \Psi^{*}\left(u^{\prime}-u\right) \cdot D\left(u^{\prime}, u^{\prime}-u\right)$.

The function $D\left(u^{\prime}, u^{\prime}-u\right)$ reveals differences between the linear information limit and the non-linear fringe limit. For linear image contributions, $u^{\prime}$ or $\left(u^{\prime}-u\right)$ is zero, the spread-of-focus TCC function collapses to the spread-of-focus envelope function $B(u)=\exp \left\{-1 / 2 \pi^{2} \lambda^{2} \Delta^{2} u^{4}\right\}$ and temporal coherence determines the upper (information) limit to linear transfer. Although spatial coherence can be a factor in determining the resolution in any single image, only temporal coherence sets the overall MIL for the HRTEM [2,3,5], since spatial coherence effects are nullified at alpha-null defocus [6]. Similarly, the finest fringe spacing in any one image can be governed by spatial coherence, however there is no overall "fringe resolution limit" set by either spatial or temporal coherence. The only limits on generating ever-finer image fringes are set by environmental factors such as horizontal vibration or test-object factors such as high-frequency falloff of atomic scattering factors [1-3]. For this reason, the use of Young's interference fringes (YIFs) to measure the MIL or MRL is flawed $[7,8,9]$, since YIFs extend to all frequencies present in the image, including those from non-linear (non-structural) contributions. Unfortunately, YIFs continue to be used to claim deceptively high HRTEM information limits [10]. This misguided practice should be strongly discouraged [11]. 
[1] M.A. O'Keefe, Ultramicroscopy 108 (2008) 196-209.

[2] M.A. O'Keefe, 37th Ann. Proc. EMSA, San Antonio, Texas (1979) 556-557.

[3] M.A. O'Keefe, L.F. Allard \& D. A. Blom, J. Electron Microscopy 54 (2005) 169-180.

[4] O. Scherzer, J. Applied Physics 20 (1949) 20-28.

[5] M.A. O'Keefe, E.C. Nelson, Y.C. Wang and A. Thust, Phil. Mag. B 81 (2001) 11, 1861-1878.

[6] M.A. O’Keefe et al., Ultramicroscopy 89 (2001) 215-241.

[7] J. Barthel \& A. Thust, Phys. Rev. Letts. 101 (2008) 200801.

[8] M.A. O’Keefe, L.F. Allard \& D. A. Blom, Microscopy \& Microanalysis 14 S02 (2008) 834-835.

[9] M.A. O'Keefe, L.F. Allard \& D. A. Blom, Microscopy \& Microanalysis 15 S02 (2009) 1470-1471.

[10] C. Kisielowski et al., Microscopy \& Microanalysis 14 (2008) 454-462.

[11] Research at Oak Ridge National Laboratory's High Temperature Materials Laboratory sponsored by U.S. Department of Energy, Office of Energy Efficiency and Renewable Energy, Vehicle Technologies program.
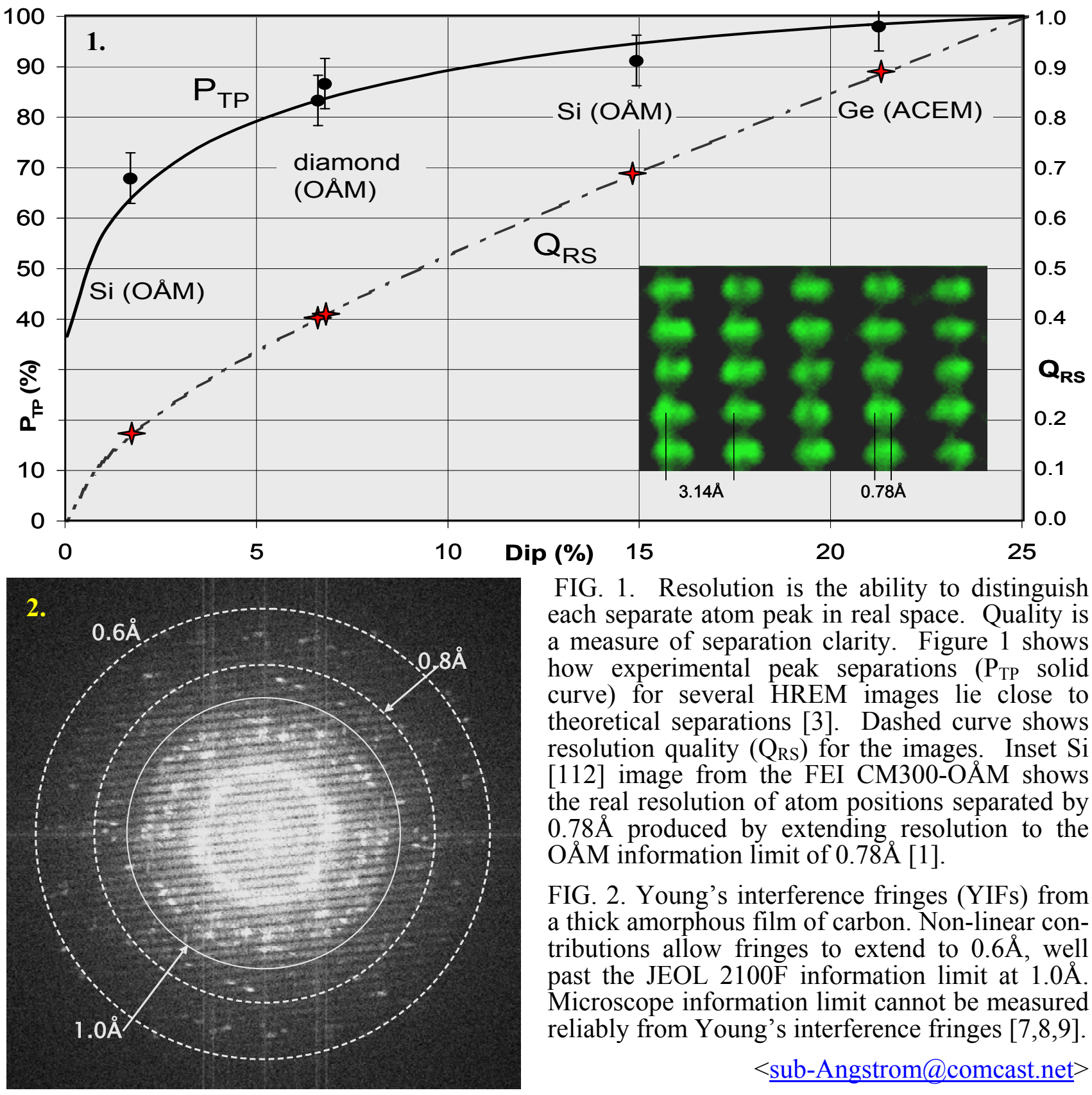

FIG. 1. Resolution is the ability to distinguish each separate atom peak in real space. Quality is a measure of separation clarity. Figure 1 shows how experimental peak separations ( $\mathrm{P}_{\mathrm{TP}}$ solid curve) for several HREM images lie close to theoretical separations [3]. Dashed curve shows resolution quality $\left(\mathrm{Q}_{\mathrm{RS}}\right)$ for the images. Inset $\mathrm{Si}$ [112] image from the FEI CM300-OÅM shows the real resolution of atom positions separated by $0.78 \AA$ produced by extending resolution to the $\mathrm{O} \AA \mathrm{M}$ information limit of $0.78 \AA$ [1].

FIG. 2. Young's interference fringes (YIFs) from a thick amorphous film of carbon. Non-linear contributions allow fringes to extend to $0.6 \AA$, well past the JEOL $2100 \mathrm{~F}$ information limit at $1.0 \AA$. Microscope information limit cannot be measured reliably from Young's interference fringes [7,8,9].

< sub-Angstrom@,comcast.net $>$ 